Daimon. Revista Internacional de Filosofía, n 75 (Septiembre-Diciembre) 2018, 105-115

ISSN: 1130-0507 (papel) y 1989-4651 (electrónico)

http://dx.doi.org/10.6018/daimon/336091

\title{
Puntos de vista y problemas de Gettier*
}

\author{
Points of View and Gettier's problems
}

ANDRÉS L. JAUME**

\begin{abstract}
Resumen: Mi objetivo es relacionar la idea de un punto de vista absoluto con los problemas de Gettier. Para lograr este propósito, tomo algunas consideraciones previas sobre las estrategias analíticas que usa Gettier y las critico apelando a algunos resultados tomados del $\mathrm{KrV}$ de Kant en su Disciplina de la Razón Pura (KrV). Finalmente, presento algunas conclusiones sobre la naturaleza del conocimiento humano y cómo podemos responder a la pregunta qué significa que $\mathrm{S}$ sabe que $\mathrm{p}$.

Palabras clave: Análisis, Definición, Epistemología, Kant, Problemas de Gettier, Puntos de vista
\end{abstract}

\begin{abstract}
My goal is to relate the idea of an absolute point of view with the problems of Gettier. To achieve this purpose, I take some preliminary considerations on the analytical strategies used by Gettier and criticize them by appealing to some results taken from Kant's Krv in his Discipline of Pure Reason. Finally, I present some conclusions about the nature of human knowledge and how we can answer the question about what it means that $\mathrm{S}$ knows that $\mathrm{p}$. Key words: Analysis, Epistemology, Definition, Kant, Gettier's Problems, Point of View
\end{abstract}

Los problemas de Gettier han dominado buena parte de la Epistemología de corte analítico y las diferentes estrategias que se han empleado para dar una respuesta a los mismos han constituido, en buena medida, el desarrollo contemporáneo de la misma centrándose de manera preferencial en el problema de la justificación epistémica. Sin embargo, el objetivo de este estudio radica en dos puntos (1) hacer explícito hasta qué punto la estrategia de análisis empleada por Gettier es acertada y (2) señalar como presupuesto básico de la misma un problema que denomino «el punto de vista absoluto». Finalmente propongo una serie de consideraciones acerca de qué es conocimiento y en qué medida la teoría de los puntos de vista puede ser una herramienta notable en el tratamiento del problema de la definición o caracterización del conocimiento.

Recibido: 30/06/2018. Aceptado: 21/10/2018.

* $\quad$ Este artículo se enmarca dentro del Proyecto de investigación PoV (Points of View) FFI2014-57409-R.

** Profesor contratado doctor en la Universidad de las Islas Baleares (andres.jaume@uib.es) Sus líneas de investigación son la Metafísica y la Teoría del Conocimiento, además de los estudios comeniológicos y la Filosofía de la Educación. Sus últimas publicaciones son: «Sobre el tomismo de Suárez», Estudios Filosóficos, LXVI, 2017, pp. 463-487 y «Ortega’s Philosophical Semantics», en Kozak \& alii (Eds.): Uses, Misuses, Abuses of Language in Culture, Literature and Linguistics, Siedlce University of Natural Sciences and Humanities-Faculty of Humanities, Siedlce, Polonia, 2018, pp. 11-20. 


\section{§1. Las consecuencias del análisis: unas reflexiones en torno a las diferentes maneras de hacer filosofía y entender el análisis}

El análisis de Gettier de S sabe que p sigue una estrategia de definición en términos de condiciones si y solo si, es decir, podemos reemplazar el analisandum por el analysans y viceversa. De forma que podemos decir que alguien sabe que p si y solo si tiene una creencia, está justificado y también es verdad. Y viceversa, sucede lo mismo si afirmo que alguien que tiene una creencia justificada y verdadera, entonces tiene conocimiento.

La situación de Gettier (1963) desacredita esta estrategia de definición. Después de considerar sus contraejemplos, nos vemos obligados a decir que las tres condiciones son solo necesarias, pero no suficientes. Además, si se aceptan las conclusiones de Gettier, se debe aceptar que se ignora todo o se está condenado al escepticismo. La aceptación de los resultados de Gettier implica que se debe tomar una decisión sobre cómo definir el conocimiento, al menos el conocimiento proposicional. Es éste el más genuinamente humano pues está ligado indisociablemente a nuestras actividades lingüísticas que han configurado que seamos lo que somos. De otra manera, toda aquella información que precisamos para nuestra vida y que conforma el lazo social que hace que no seamos simples mónadas sino seres interdependientes los unos de los otros, radica en el lenguaje. De forma que, si queremos saber que es el conocimiento, debemos lograr un análisis del enunciado $\mathrm{S}$ sabe que $\mathrm{p}$.

Ahora bien ¿en qué consiste elaborar tal análisis? Claro está que Gettier se mueve en el ámbito de la filosofía analítica que, en un momento dado de la historia, se planteó como el otro gran continente filosófico respecto a lo que se denominó Filosofía continental. A finales del siglo pasado, salvo en determinados círculos muy ortodoxos, ya no tenía sentido hablar de tal dicotomía. La postmodernidad y la globalización licuaron ambas categorías. No obstante, entre los llamados filósofos analíticos persiste la idea de que hacer filosofía es cuestión de análisis (como entre los de corte fenomenológico reaparecen constantemente conceptos como «reducción», «epojé» o «esencia»). La pregunta, es, pues, en qué consiste ese proceder analítico.

La llamada Filosofía analítica ha tenido diversas concepciones acerca del análisis, en caso de que se considere a éste como el rasgo definitorio de esa misma corriente, algo que, según Glock (2012) dudosamente puede afirmarse ${ }^{1}$. Ha habido diversas maneras de entender el análisis a lo largo de la historia de esta manera de hacer filosofía y, en algunos casos, incluso incompatibles. No es lo mismo proceder a un análisis descomposicional como proponía Moore -descomposición de conceptos en conceptos máximamente simples- o las estrategias seguidas por B. Russell, en su teoría de las descripciones definidas, y el Wittgenestein del Tractatus, que parecían buscar los átomos lógicos, bien fueran datos sensoriales irrefutables -no cayeron en la cuenta del mito de lo dado- o bien átomos metafísicos, que seguir la estela de Austin y la filosofía oxoniense centrada en el lenguaje corriente y no en la idea de que hay un lenguaje ideal ocultado por el uso lingüístico habitual. Son presupuestos

1 «En consecuencia, mientras que las definiciones más pesadas y específicas del análisis ya no cubren todo el ámbito de la filosofía analítica, las menos exigentes y más amplias son demasiado indiscriminadas. El análisis, por tanto, no podrá ser usado para definir la filosofía analítica.» Glock, H.J.,(2012), ¿Qué es la filosofía Analítica?, Madrid: Tecnos, p. 201. 
de fondo que importan, porque la práctica del análisis e incluso lo que se espera de él es siempre dependiente de ese tipo de presupuestos-marco sobre los que se teje una determinada manera de hacer las cosas.

En cualquiera de los casos, tanto Moore como Russell y el primer Wittgenstein parecieran tener detrás la metáfora del químico aislando los componentes de sus substancias a través de un refinado proceso de análisis para identificar de qué están hechas.

Obviamente la idea de análisis de los arriba citados estaba ligado a una tesis más fundamental, si había que analizar era porque el lenguaje común ocultaba de alguna manera el problema que deseaba verse con claridad, una idea en absoluto nueva ${ }^{2}$ que encontramos ya en el s. XVII -i.e. Comenius-.

En lo que respecta a filósofos como Gettier o Grice está claro que no buscan algo así como los conceptos últimos de la realidad como propusiera Moore, pero sí hay una búsqueda de las condiciones necesarias y globalmente suficientes para decir que un término significa lo que significa. Dentro de esa corriente, en el terreno de la Epistemología o Teoría del conocimiento el ejemplo por excelencia lo constituye el artículo de Gettier de 1963. Dar con ese tipo de análisis consistía básicamente en dar una definición, pero ¿qué tipo de definición?

Se han considerado muchas maneras de definir un término: definiciones denotativas o extensivas, es decir, dar una serie de ejemplos de los objetos denotados por el término, pese a que el conjunto en sí sea infinito -i.e. los números naturales- o prácticamente innumerable -i.e. como sucede con términos como «flor»o «estrella». Otra técnica definitoria es la ostensión, es decir, cuando decir «esto es X» o cuando en la misa el sacerdote eleva la ostia y el cáliz y dice que es el «cordero de Dios», de hecho este último ejemplo no es trivial pues un «ostensorio», según la RAE es el objeto donde se hace ostensión de algo, es decir, donde manifiesta algo, y ese algo es lo que es. Sin embargo, ese tipo de definiciones corren el riesgo de no ser más que otra variedad del mito de lo dado o, en términos del Wittgenstein de la Investigaciones, una variedad de lenguaje agustiniano que nada convence como manera de dar el significado de un algo. Bien sabemos que por mucha ostensión que hagamos del cuerpo de Cristo, un ateo no verá absolutamente nada, como no supieron distinguir los primeros indios americanos a los españoles de sus caballos ${ }^{3}$.

Igualmente tenemos las definiciones por sinonimia, pero ya nos advirtió Quine de la oscuridad de semejante noción en Dos dogmas del empirismo. La sinonimia es poco precisa y cualquiera que haya estudiado lenguas -vivas o muertas- y no meramente lenguajes formales, sabe que no hay sinónimos perfectos.

También disponemos de definiciones operacionales, muy usadas en determinadas ciencias. En una definición operacional el definiens alude a una operación pública y repetible. Las definiciones operacionales de un término nos dicen que la relación entre definiendum y definiens es bicondicional y que el definiens es algún tipo de operación especificada de antemano. Por ejemplo, «X es una bacteria gram negativa syss $\mathrm{X}$ da negativo en la tinción de Gram» o «S padece hipertensión arterial syss los valores de la tensión sistólica y diastólica son superiores a 130/90 mmHg caeteris paribus $\mathrm{S}$ no toma medicación antihipertensiva (betabloqueantes, inhibidores de la angiotensina, etc). Obviamente esto no parece que sea

2 Cf. Eco, U. La búsqueda de la lengua perfecta, (2015), Barcelona: Crítica, pp. 178-192.

3 Cf. Eco, U., Kant y el ornitorrinco, Barcelona: Debolsillo, 2013, p. 171-173. 
una técnica aplicable en filosofía, donde quizás, sólo tendría lugar en el uso de términos de nuevo cuño como el de «función propia» que emplea Ruth Millikan y que, por supuesto, ha sido ampliamente discutido y que muy bien podríamos clasificar dentro de la categorías de definiciones reales, es decir, tanto el definiens como definiendum son equivalentes y refieren a la misma cosa, de forma que el definiens se constituye como análisis del definiendum. Este tipo de definiciones son muy usadas en ciencias experimentales o naturales y no menos en determinadas corrientes filosóficas que buscan algo así como las esencias de los fenómenos que estudian, de ahí que la definición de función propia sea una definición real en el sentido de que remite a una realidad a la que no tenemos un acceso operacional sino que simplemente tratamos de captar en tanto que análisis de un contenido en sus aspectos distintivos. Cohen y Nagel (1976) señalan que entran en este ámbito también las matemáticas y apuntan al siguiente ejemplo de lo que consideran una definición real:

«La figura A es semejante a la figura A'. =. La razón de la distancia entre dos puntos cualesquiera , P y Q, de A y la distancia entre los puntos correspondientes , P'y Q' de A', es constante. Df.»

Obsérvese que la definición es extensional, es decir, admite substituciones salva veritate, de modo que podemos sustituir el definiendum por el definiens en cualquier momento. Con este tipo de definiciones delimitamos el concepto en cuestión. La alternativa sería la definición verbal o nominal, a saber, el acuerdo o resolución en lo relativo al uso de signos verbales (Cohen \& Nagel, 1976, p. 47), son ejemplos las tautologías de la lógica del tipo p implica q syss no p o q; de modo semejante $\mathrm{a}^{2}=\mathrm{a} \cdot$ a por definición. Sostienen Cohen y Nagel que las definiciones nominales, a diferencia de las definiciones reales no pueden ser verdaderas o falsas, son materia de una resolución. Es difícil aclarar los límites en la práctica, de modo que las definiciones nominales a menudo hay referencias a lo que los términos representan.

Podemos hacer muchas y variadas taxonomías de los diferentes tipos de definición. Lo que interesa ahora es vincular la noción de definición a la de análisis en la medida en que entendió el análisis filosófico Gettier. La pregunta que debemos formularnos es qué tipo de definición se ejemplifica en el análisis de Gettier. Todo parece indicar que, de algún modo, se trata de una definición nominal. Aunque resulta extraño reducir el problema del conocimiento a un simple asunto de toma de decisiones o resoluciones acerca de lo que el término significa.

Tras los contraejemplos de Gettier se desliza algo así como la siguiente afirmación «Si aceptáramos la definición tripartita y la aplicáramos a los casos que se presentan, nadie en su sano juicio estaría tentado a sostener que ambos sujetos conozcan p». El conocimiento no puede ser un mero acuerdo o resolución acerca de las palabras sino que se le exige una realidad, lo que se pone en juego con el conocimiento no es poca cosa, es la fiabilidad de nuestras acciones sustentadas en conocimientos genuinos, de ahí que se diga que el objeto del conocimiento es la verdad. En ese sentido creo que Gettier se aparta de la idea de una posible definición nominal muy del gusto de aquellos filósofos que quisieron ver sólo problemas dentro de la selva del lenguaje y malentendidos verbales que debían aclararse mediante alguna estrategia analítica más o menos sofisticada, es decir, debíamos sacar a la 
mosca de su botella, pero ¿quería salir la mosca? ¿realmente estábamos encerrados en una botella lingüística que se nos interponía como velo ante la realidad misma? Lo dudo. Sea como fuere, no parece que sea tarea fácil clasificar el intento de Gettier y no nos quede otra opción que contentarnos con la pobre respuesta «Gettier trata de lograr un análisis de la expresión $\mathrm{S}$ sabe que $\mathrm{p}$ teniendo en cuenta una idea de sentido común acerca de qué significa asentir ante $S$ sabe que p».

La definición de conocimiento que propusiera Gettier en 1963 -en realidad una reelaboración muy cuestionable de la tercera parte del Teeteto de Platón (201 c-d)- consigna que ofrecer un análisis de un término es, ni más ni menos, una cuestión de estipular condiciones syss entre definiendum y definiens. Respecto de los problemas de Gettier, problemas que han condicionado en buena parte la epistemología analítica desde 1963 hasta nuestros días -ya sea explícita o veladamente-, la Filosofía Analítica ha ensayado diferentes enfoques, como la búsqueda de una cuarta condición misteriosa o la reformulación completa de los mismos, centrándose en el concepto de justificación epistémica. Hasta que, según algunos, como Glock (2012) han apuntado que «la actual tendencia consiste en renunciar a una definición analítica y concentrarse más en el papel que juega el concepto de conocimiento en nuestras prácticas» (p. 200). Es decir, para Glock el problema del análisis de Gettier se ha disuelto como un azucarillo en agua. Sin embargo, considero que, pese a la renuncia a tales tipos de análisis, sí persiste una tendencia, quizás una vuelta atrás velada, a lo que desde antaño se denominó «criteriología» y que no es otra cosa que plantearse qué es la justificación epistémica y en qué se diferencia de la simple creencia. De otra manera, no analizaremos según el proceder de Gettier, pero sí especularemos -ésa y no otra es la palabra- acerca de qué consiste dar buenas razones y qué límites tienen esas en la vida humana que, respecto del conocimiento, es la única que más o menos nos resulta próxima. Obviamente no haremos de la filosofía un saber analítico en ese sentido, pero sí un saber discursivo.

La cuestión es si eso es un procedimiento adecuado para el tratamiento filosófico de determinados problemas o, por el contrario, lo es para otras disciplinas como la Matemática o el Derecho.

\section{§ 2. ¿Por qué deberíamos cambiar las reglas del juego?}

El problema de Gettier se basa en una de metáfora que en mi opinión no ha sido adecuadamente considerada. Se trata de algún modo de una variante de lo que Kant señala a propósito de la diferencia entre las matemáticas y la Filosofía en la KrV.

La segunda parte de KrV es la parte menos estudiada. Körner, Gardner y otros filósofos analíticos interesados por Kant no «analizan» esta parte. Sin embargo, el método es la pregunta que preocupa una gran parte de la reflexión teórica en la filosofía moderna y contemporánea, desde Galileo a Popper, Kuhn o Lakatos. Tener un método es una forma de obtener justificación epistémica. Si sigo un conjunto de reglas bien establecidas, es decir, reglas de inferencia, entonces es cierto que pueda dar con el pretendido conocimiento. Ya sean estas reglas razonamientos deductivos, inductivos, abducciones o razonamientos estadísticos. Lo cierto es que tienen cierta confiabilidad para obtener buenos resultados que dicen que son verdades. 
La cuestión del método es desarrollada por Kant en una disciplina -regulaciones negativas o restricción de la razón- y reglas positivas -Canon de la Razón Pura-. Pero, a pesar del trascendentalismo y otros compromisos importantes, me centraré en la primera argumentación de la Disciplina de la razón pura (A731 / B741 y ss.). De hecho, en este capítulo 1, Kant considera la relación entre las Matemáticas y la Filosofía y establece claramente cuál es la diferencia. La filosofía y las matemáticas usan conceptos, pero de una manera diferente. Mientras que las matemáticas construyen conceptos, la filosofía expone los conceptos de una manera discursiva. Los lectores pueden sorprenderse con la idea de la construcción y, probablemente, estar insatisfechos con la definición que da Kant (A713 / B741-A714 / B742). Sin embargo, el problema no surge aquí, sino en A727 / B755 y ss. En resumen, después de afirmar que la Filosofía consiste en un conocimiento de sus propios límites, sostiene las siguientes tesis:

1. La filosofía no tiene definiciones de la misma manera que las matemáticas. La filosofía ofrece definiciones como exposiciones de conceptos dados, es decir, una definición filosófica es «un ensayo» (A730 / B758) y, en consecuencia, la Filosofía no debe imitar a las Matemáticas.

2. No tenemos axiomas ni teoremas.

3. No tenemos demostraciones como las matemáticas, que poseen demostraciones apodícticas, trabajamos con pruebas acroamáticas (un dispositivo discursivo). Acroamático significa una manera de enseñar por medio de discursos, narraciones o explicaciones, una cosa diferente es lo que es una explicación, quizás una fábula o una analogía con cierta verosimilitud). Sin embargo, Kant incide en la discursividad y no en explicaciones o narraciones.

En absoluto estamos obligados a seguir los dictados de Kant, pero sí es obvio que nos alertan acerca del peligro de buscar definiciones como si estuviéramos haciendo matemáticas. Es decir, si por algo se caracteriza la filosofía es porque, ante todo, es una actividad discursiva que, como decía Platón en la República (511 c) era un proceder de las ideas, a través de ideas y que llega a ideas. Cuando hacemos filosofía simplemente realizamos una actividad que se constituye en el lenguaje como mediación intersubjetiva básica y que tiene por resultado una puesta a punto de los conceptos con los que nos enfrentamos a la realidad. ¿Buscamos la verdad? sí, pues la verdad es el objeto de toda empresa epistemológica en principio, otra cosa será que incluso el concepto de verdad sea objeto de discursividad y debamos aclararnos acerca de qué cuenta como verdad y, más importante, por qué. Pero son esos temas que deben ser tratados en otro momento.

En lo que respecta a la concepción del análisis de Gettier es algo que no cabe desdeñar. La metáfora matemática tiene su origen en la polémica acerca de los fundamentos de las matemáticas y, particularmente, en la visión de Russell sobre cómo fundamentar las matemáticas. El argumento que Russell parece responder es la idea de que la lógica es una cuestión de analiticidad, mientras que la matemática es una cuestión de juicios sintéticos a priori. Pero si podemos reducir las matemáticas a la lógica, las matemáticas también son una cuestión de juicio analítico y todos saben lo que implica tener un juicio/proposición analítica. De esta manera, la Filosofía es también una cuestión de análisis y, en consecuencia, esta es 
la vía del conocimiento filosófico. Tenemos ejemplos clásicos de diferentes aplicaciones de este método, es decir, la teoría de descripciones definidas, el análisis del significado dado por Grice o la propuesta comentada anteriormente y desarrollada por Gettier. En resumen, realizar el análisis de un concepto es lo mismo que definir en términos de las condiciones syss entre analysandum y analysans.

Estas estrategias tienen éxito en campos como el Derecho y las Matemáticas, de hecho, podemos observar cómo, de alguna manera, el jurista y el matemático construyen ${ }^{4}$ sus conceptos, en algún sentido muy lato y distante, quizás, de lo que Kant entendiera por construcción.

Considérese la noción de matrimonio como institución social y legal. El código civil, en el derecho continental, hace positiva una práctica social considerando algunos parámetros y especificándolos. En pocas palabras, determinada institución jurídica es matrimonio syss tiene algunas condiciones establecidas explícitamente. Fuera de estas condiciones, no hay matrimonio. Aunque no todos los conceptos jurídicos son tan claros en su aplicación -i.e. el delito de daños contra el honor-, determinadas instituciones civiles como el matrimonio o la ciudadanía aparecen muy claramente definidas en el texto legal. De hecho nos encontramos que una cosa es la aplicación del concepto jurídico, otra su definición y otra su problematización con otros conceptos jurídicos. En cualquier caso lo que sí podemos afirmar es que ha habido una construcción, es decir, no sólo hemos positivizado una práctica social y la hemos sancionado a través del derecho, sino que, en cierto modo, hemos creado un concepto para una práctica que, primero nebulosa, ahora ya menos, se ha definido en términos constructivos. Aquí sí funciona una definición en términos de syss, pero ¿funciona con términos como «bondad» o «conocimiento»?

La pregunta ahora es si los conceptos filosóficos funcionan de esta manera, si construimos conceptos o, de lo contrario, recogemos términos del discurso ordinario que se pueden definir de diferentes maneras y bajo diferentes criterios. En una palabra, el pluralismo es la norma y no la excepción en el discurso filosófico y la pregunta es si somos capaces de tal empresa para hacer filosofía. no parece que con términos como bondad o conocimiento podamos emplear la misma estrategia, más bien serán definiciones a modo de ensayo como Kant señala. ¿Qué quiere decir esto de «a modo de ensayo»? Significa que no aspiramos a la precisión y limitación que impone la estrategia syss y que, en cambio, nos decantamos por una condiciones más borrosas, que conllevan un tanteo entre el uso de las palabras dentro de la comunidad lingüística y en relación con el mundo extralingüístico, a saber, la cuestión del ensayo o tanteo se dirime en ¿funciona o no funciona el concepto para mis propósitos? Y los propósitos no son otra cosa que teleología, algo funcionará para un determinado propósito

4 En un sentido alejado de la idea de construcción que emplea Kant a propósito de las matemáticas, Glock refiere a los procedimientos de Carnap o Quine como construccionismo lógico. A este respecto señala Glock lo siguiente: «En esta parcela de la filosofía analítica, el análisis no es la descomposición en sus componentes de un complejo dado, sino que es más bien un acto de construcción. Así pues, tanto para Carnap como para Quine, análisis significa «explicación lógica». Su objetivo no es la aportación de un sinónimo del analysandum, o incluso de una expresión con las mismas condiciones necesarias y suficientes de aplicación. Tampoco consiste en la identificación de los verdaderos constituyentes y formas que él mismo posee bajo la superficie gramatical. Su objetivo es más bien el de suministrar una expresión alternativa o construcción que ayude igualmente a los objetivos cognitivos del original, mientras evita sus inconvenientes científicos o filosóficos (...).» Glock, H.J., ¿Qué es la Filosofía Analítica?, Madrid: Tecnos, 2012, p. 200. 
si cumple con las expectativas que ya nos vienen dadas en un marco conceptual previo. De esta forma ensayar un concepto como el de «flogisto» en el marco científico actual parece ser una empresa abocada al fracaso, al igual que un término como «forma substancial»o «substancia espiritual», pero no uno como «bien», «justicia» o «verdad», por mucho que se hable de la postverdad.

\section{§3. Los problemas de Gettier, justificación epistémica y Puntos de Vista}

La teoría de los puntos de vista tiene una larga historia ${ }^{5}$. La formulación que emplearemos aquí procede en buena medida de Vázquez \& Liz (2011) y puede definirse de la siguiente manera: Un Punto de vista o PdV tiene la siguiente estructura ${ }^{6}$ :

$$
\mathrm{PdV}=\langle\mathrm{P}, \mathrm{R}, \mathrm{n}-\mathrm{CC}, \mathrm{CC}, \mathrm{Cp}\rangle
$$

Donde $\mathrm{P}$ es el portador, $\mathrm{R}$ el conjunto de relaciones que conecta al portador $\mathrm{P}$ con los contenidos que se denotan de la siguiente forma n-CC el contenido no conceptual, $\mathrm{CC}$ el contenido conceptual. Finalmente $\mathrm{Cp}$ denota las condiciones de posesión de un PdV. Puede decirse, de manera intuitiva que un $\mathrm{PdV}$ es un sistema que conecta o relaciona contenidos con un portador (Hautamaki 2016, p. 508). Igualmente Hautamaki (ibíd., p. 494) señala la ambigüedad del término, aunque prefiero apuntar a una laxitud que permite adaptar la noción de PdV a los intereses filosóficos de quien emplea el concepto. De ahí que haga las siguientes consideraciones: (1) El portador puede ser, a mi juicio, tanto un sujeto particular como un sujeto colectivo. (2) Cuando el sujeto del PdV es colectivo y suficientemente amplio -i.e. una generación o un grupo de ellas- lo denominaré sin más «marco» y añadiré que los marcos son sistemas de creencias colectivas que muy bien pueden satisfacer el esquema propuesto por ambos. (3) Podemos considerar también un PdV absoluto. Así, frente a la parcialidad que parece inducir el término, introduciré como concepto de nuevo cuño la idea de PdV absoluto que, en realidad, es la negación de los PdV o la suma total de todos ellos; lo primero si lo que queremos negar el perspectivismo intrínseco a la noción de PdV y, lo segundo, si lo que queremos entender es que el PdV absoluto asume toda la parcialidad y, por lo tanto, todo lo ve, no en escorzo sino tal como es.

La idea de PdV particular, y PdV absoluto creo que deben ser tenidas en cuenta a la hora de revisar las propuestas de Gettier. Mucho se ganará si consideramos su análisis desde esta perspectiva.

Gettier sugiere que no tenemos una justificación completa de nuestros motivos para afirmar que $\mathrm{S}$ sabe que p. Este es el núcleo del argumento de Gettier contra la definición tripartita, sin embargo, no se olvide que nada se discute acerca de la verdad. Es decir el análisis de la definición tripartita se mueve en el terreno de la pura lógica y lo que quiere poner de manifiesto es que el azar nos puede jugar una mala pasada. De forma que los dos metaproblemas a los que nos debemos enfrentar son: (1) el azar, a mayor incertidumbre,

5 Cf. Vázquez, M. \& Liz, M. (2015), Temporal Points of View: Subjective and Objective Aspects, Springer, Chap. 1.

6 Cf. Vázquez, M., \& Liz, M. (2011), « Models as points of view: The case of system dynamics», Foundations of Science, 16(4), 383-391. 
menor conocimiento. Problema que posteriormente desarrollaría Dretske (1981) como intento de respuesta a Gettier en su idea de relacionar el conocimiento con la teoría de la información. (2) La justificación parece que tiene que ver con realizar inferencias correctas $\mathrm{y}$, sin embargo, pese a que realicemos inferencias correctas, no tenemos suficiente justificación. (3) El conocimiento y la verdad son absolutos. Si la verdad no fuera absoluta entonces violaríamos el principio de tertium non datur -como señaló Russell en 1905 para justificar su teoría de las descripciones definidas- y, si nos movemos en el pantanoso y difuso terreno de la verdad o la justificación parcial, siempre habrá un resquicio para el azar.

Sostendré que (3) es la opción a la que me voy a plegar, en pocas palabras, no tengo acceso al ojo de Dios y he ahí la suposición oculta que quiero señalar en el análisis del conocimiento que, a la vez justificará el tratamiento que hago del problema desde la noción de Punto de Vista.

En mi opinión, hay una suposición oculta en el argumento de Gettier. Alguien sabe muy bien cómo es la situación real, es decir, hay un observador externo que puede afirmar «los hechos son de esta manera». Además, parece que este observador no es falible o, de lo contrario, omnisciente. Entonces, diferencio el punto de vista de los dos personajes en los acertijos de Gettier o el observador que afirma por mera casualidad qué hora es desde el punto de vista de nuestro observador omnisciente. Los primeros observadores tienen un punto de vista particular y están convencidos de que tienen razón. Ése es el presupuesto de los dos casos de Gettier en 1963 y Gettier habla como si se diera un punto de vista externo que ve cómo son las cosas. Si ese PdV fuera otro sujeto humano, ¿quién nos aseguraría su no falibilidad o que el azar no se cebaría con él? Por lo tanto debemos suponer que el observador omnisciente tiene un punto de vista absoluto. Sostendré que este punto de vista absoluto es el de Dios, el ojo que todo lo ve, además, como dijeron los jesuitas en la polémica De Auxiliis, este Dios tiene Scientia media, es decir, puede ver pasado, presente, futuro y los futuribles, o de otra manera, Él conoce la necesidad y la contingencia de cada evento en el mundo incluso de aquellos que, como las acciones humanas, no están determinados.

$\mathrm{X}$ es un punto de vista absoluto syss $\mathrm{f} \mathrm{X}$ sabe que $\mathrm{p}$ es necesario o es contingente; p está en correspondencia con hechos reales.

Consideraré este Punto de Vista Absoluto de Dios como una idea regulativa que garantiza una hipotética objetividad de todas las afirmaciones sobre la realidad. Obviamente, el concepto de «idea regulativa» proviene de Kant y, de hecho, Dios es la síntesis de la síntesis, es decir, la idea regulativa suprema. Sin embargo, tomo este concepto en un sentido diferente, es decir, solo aplicado por el momento a la Epistemología. En una palabra, como una forma de ofrecer alguna restricción a los diferentes puntos de vista tomados en un sentido relativista o relacional.

Si nuestras capacidades cognitivas son falibles, si la subsanación del error es algo que depende de otro ser falible tal como aparece en los contraejemplos de Gettier de manera tácita, entonces o bien vemos que jamás vamos a tener una justificación definitiva de una vez por todas y que no nos queda humanamente otra salida más que movernos en un ámbito de creencias etiquetadas provisionalmente de «conocimientos» en virtud de criterios no menos 
falibles o, por el contrario, admitimos que algo hay ahí fuera como una situación objetiva que se me impone y que esa objetividad descansa en una idea: alguien ve las cosas tal como son y, por lo tanto hay un canon, extrahumano sí, pero que, como idea regulativa, permite asegurar la certeza de que hay una objetividad que, por muchas perspectivas que tengamos o múltiples aproximaciones a la misma, está ahí y que, en última instancia es lo que hace que revisemos una y otra vez nuestros criterios de justificación de nuestras creencias, simplemente porque no siempre las cosas salen como queremos.

\section{§4. Algunas conclusiones sobre el concepto de conocimiento a partir de la teoría de los PdV}

Hasta ahora he tratado de aclarar la espinosa cuestión del análisis en general en sus relaciones con el problema de la definición. Como se ha visto, los resultados son un tanto desesperanzadores si lo que se buscaba era una clarificación definitiva y unánime del problema. El problema persiste y mucho tendrá que ver su curso cual enfermedad crónica con la decisión que se adopte.

En lo tocante a los problemas de Gettier el objetivo no ha sido tanto ofrecer una solución -no creo que la haya- como explicitar aquellos aspectos de su formulación que he considerado necesarios para su comprensión tales como la idea de un punto de vista absoluto, con ello afirmo que el saber no es cuestión baladí por mucho que de construcción social tenga, de otra manera, no podemos inventarnos el saber, pero sí estamos condenados a innovar nuevas estrategias en lo que desde Nicolás de Cusa se denomina venatio sapientiae. Aún así, he considerado oportuno tratar la cuestión de los puntos de vista como estrategia para abordar el problema del conocimiento. Dicho esto, no me queda otra cosa que hacer una serie de afirmaciones programáticas acerca de una hipotética pars construens acerca de la naturaleza del conocimiento. Entre mis tesis substantivas señalaría las siguientes:

1. El saber se estructura en marcos de referencia, es decir, el saber cuenta como saber o conocimiento dentro de una estructura que denominaré marco y que es análoga en buena medida a un punto de vista colectivo.

2. El conocimiento humano es limitado, falible y dado en perspectiva. Por lo tanto, la justificación epistémica también es limitada y no definitiva. La cuestión del perspectivismo es fundamental si lo que se quiere lograr -quizás a lo único que podamos aspirar- sea un conocimiento provisional que ofrezca seguridad, aunque no invulnerabilidad. Este tipo de conocimiento es el que nos encontramos en actividades como la ingeniería, la medicina o la agricultura. El azar siempre representa una amenaza pero la obligación humana es tratar siempre de poner un cerco a ese mismo azar y, en consecuencia, no queda otra opción más que ir asegurando y revisando constantemente nuestras prácticas epistémicas. La idea de que se actúa desde un determinado PdV nos hace más conscientes de la falibilidad de nuestros sistemas de creencias y, en consecuencia, la necesidad constante de revisión.

3. El saber se estructura por medio de marcos es decir, hay constructivismo (cognitivo y social), pero este concepto no debe entenderse como un sinónimo de invención. Un 
concepto, el elemento básico del conocimiento que solo tiene sentido en el juicio es una ficción heurística (KrV A771/ B799) y como tal nos ayuda a manejarnos entre las cosas.

4. El conocimiento tiene una dimensión pragmática, en otras palabras, los ignorantes perecen. Conocer es una exigencia vital que garantiza nuestra vida, es una necesidad fundamental.

5. La verdad es el objetivo de nuestras prácticas epistémicas. Sostengo por el momento una noción de Verdad como S-asertabilidad o asertabilidad de un enunciado en un lenguaje L (Sellars, 1991, p. 177 y ss.) a lo que habría que añadir la dimensión intersubjetiva y conformadora de marco que tiene el lenguaje. Además, los marcos no son invenciones o ficciones puras, sino ficciones pragmáticas que funcionan mientras no se demuestre lo contrario. Si fallan, estamos obligados a volver a fijar o cambiar en cierto grado nuestro marco (La Historia de la Ciencia y o de la cultura científica ofrece excelentes ejemplos) hasta que demos con resultados satisfactorios, de ahí que todo saber o práctica epistémica tenga unos criterios de valoración y se adapte a un determinado ethos o forma de vida.

6. Los marcos tienen una porción de conjetura y una porción de herencia. El último es el primero, es decir, el conocimiento es conservador y se da por grados. Que es conservador significa que retenemos aquellos resultados que se han mostrado útiles en el pasado y que no suscitan ninguna necesidad de cambio en la circunstancia actual. Así, por ejemplo encalar las paredes o limpiar el suelo con lejía o construir alcantarillado en las ciudades son resultados valiosos que tienen mucha historia detrás y los mantenemos a día de hoy, del mismo modo que pese a la existencia de GPS nadie se embarca sin brújula, sextante, compas y demás instrumentos de navegación. El barco de Neurath debe seguir a flote.

\section{Referencias}

Cohen, M. \& Nagel, E. (1976), Introducción a la lógica y al método científico 2. Lógica aplicada y método científico, Buenos Aires: Amorrortu.

Dretske, F. (1981), Knowledge and the Flow of Information, Cambridge, Mass.: The M.I.T. Press.

Eco, U. (2015), La búsqueda de la lengua perfecta, Barcelona: Crítica.

Eco, U., (2013), Kant y el ornitorrinco, Barcelona: Debolsillo.

Gettier, E. (1993), «Is Justified True Belief Knowledge?», Analysis, v. 23, pp. 121-123.

Glock, H.J., (2012), ¿Qué es la Filosofía Analítica?, Madrid: Tecnos.

Hautamaki, A. (2016), «Points of View: A Conceptual Space Approach» Found Sci., 21, 493-510.

Sellars, W. (1991), Science, Perception and Reality, Atasadero, California: Ridgeview Publishing Company.

Vázquez, M. \& Liz, M. (2015), Temporal Points of View: Subjective and Objective Aspects, Springer, Chap. 1.

Vázquez, M., \& Liz, M. (2011), «Models as points of view: The case of system dynamics», Foundations of Science, 16(4), 383-391. 
